\title{
The efficacy and safety of anti-interleukin- 6 receptor monoclonal blockade in a renal transplant patient with Castleman disease: early post-transplant outcome
}

\author{
Masatoshi Matsunami i* D, Yoshifumi Ubara ${ }^{2,4}$, Keiichi Sumida², Yoichi Oshima², Masahiko Oguro², Kazuya Kinoshita', \\ Kiho Tanaka', Yuki Nakamura', Keiichi Kinowaki ${ }^{3}$, Kenichi Ohashi ${ }^{3,5}$, Takeshi Fujiii, ${ }^{3}$ Takuro Igawa ${ }^{6}$, Yasuharu Sato ${ }^{6}$ \\ and Yasuo Ishii ${ }^{1}$
}

\begin{abstract}
Background: Multicentric Castleman disease (MCD) is an uncommon lymphoproliferative disease characterized by systemic inflammatory reactions associated with the dysregulated production of interleukin-6 (IL-6). In patients with $M C D$, renal involvement is uncommon, with only one report published regarding kidney transplantation (KTx) to treat end-stage renal disease (ESRD) secondary to MCD. Recent clinical observations have shown that IL-6 production is implicated in allograft rejection, while IL-6 receptor blockade (with tocilizumab [TCZ]) reduces alloantibody generation and thereby improves graft survival; however, the efficacy and safety of TCZ in MCD patients undergoing KTx is still unknown.

Case presentation: Herein, we describe the case of a 50-year-old man with MCD who received living-donor KTX for ESRD. Post-operative immunosuppression consisted of a triple-drug regimen (tacrolimus, mycophenolate mofetil and methylprednisolone) with TCZ that was administered intravenously every 2 weeks. At 17 months post-transplantation, the patient remains asymptomatic, and the allograft pathology has shown no evidence of rejection and no development of de novo donor-specific antibody (DSA).

Conclusions: To our knowledge, this is the second reported case of an MCD patient with ESRD who underwent successful KTX. TCZ safely supported the patient during the perioperative period, and this drug may be useful for blocking the generation of donor-specific antibodies and reducing the risk of rejection episodes. KTx in combination with TCZ is thus considered a viable treatment option for ESRD due to MCD.
\end{abstract}

Keywords: Castleman disease, Kidney transplantation, Tocilizumab, IL-6, IgA nephropathy

\section{Background}

Castleman disease $(\mathrm{CD})$ is an uncommon heterogeneous group of lymphoproliferative disorders with three histological types (hyaline vascular type, plasma cell type, and mixed type). $\mathrm{CD}$ can occur in a localized (unicentric CD: $\mathrm{UCD}$ ) or widespread (multicentric CD: MCD) form. The aetiology of $\mathrm{CD}$ is not well understood; however, excessive production of interleukin 6 (IL-6) by lymph node

\footnotetext{
* Correspondence: matsunami-m@toranomon.gr.jp

${ }^{1}$ Department of Surgery, Nephrology Center, Toranomon Hospital, 2-2-2

Toranomon, Minato-ku, Tokyo 105-8470, Japan

Full list of author information is available at the end of the article
}

hyperplasia has been implicated in its pathogenesis [13]. The clinical features include generalized lymphadenopathy, organomegaly, and systemic manifestations, such as fatigue, a low-grade fever, and weight loss. Abnormal laboratory findings include anaemia, hypoalbuminaemia, hypocholesterolaemia, hypergammaglobulinaemia, and increased levels of C-reactive protein (CRP) [1-3]. Renal involvement in MCD seems to be uncommon and has been described in only a few case series $[4,5]$. To date, there has been only one report of kidney transplantation (KTx) for the treatment of end-stage renal disease (ESRD) secondary to MCD [6].

(c) The Author(s). 2018 Open Access This article is distributed under the terms of the Creative Commons Attribution 4.0 International License (http://creativecommons.org/licenses/by/4.0/), which permits unrestricted use, distribution, and 
IL-6 is a key cytokine that impacts the development and maturation of $\mathrm{T}$ cells, $\mathrm{B}$ cells, and antibody producing plasma cells. Excessive IL-6 production has been linked to several human diseases characterized by unregulated antibody production and autoimmunity, such as $\mathrm{CD}[1,2]$. Tocilizumab (TCZ; Actemra ${ }^{\oplus}$, Roche/Genentech, San Francisco, CA, USA) is a first-in-class humanized monoclonal blockade targeting the IL-6 receptor (IL-6R). TCZ binds to both soluble and membrane-bound forms of the IL-6R and is approved for the treatment of CD $[1,2]$.

A recent study of TCZ for patients undergoing KTx showed a significant reduction in donor-specific antibody (DSA) production and improvement in the transplantation graft survival $[7,8]$. IL-6R interactions have been shown to be critical for alloantibody generation in an animal model of alloimmunity. IL- 6 modulates T cell immunity and is a powerful stimulant of pathogenic IgG production, so the blockade of these interactions with an anti-IL-6R monoclonal blockade results in significant reductions in alloantibodies $[7,8]$. However, the efficacy and safety of TCZ in MCD patients undergoing KTx is still unknown. To answer this question, we herein report the second known case of an MCD patient with ESRD who successfully underwent living-donor KTx using TCZ.

\section{Case presentation}

A 33-year-old man visited an outpatient clinic with haematuria and proteinuria. One year later, the patient was diagnosed with IgA nephropathy by a renal biopsy and was treated with diet and medication. At 36 years of age, his began to experience fatigue and weight loss. He was had generalized lymphadenopathy, hepatosplenomegaly, and elevated CRP levels, and his IL-6 level had gradually increased. Chest CT revealed slight enlargement of the mediastinal lymph nodes, centrilobular nodules, thickening of the bronchovascular bundles, and ground-glass opacities. These clinical findings were consistent with MCD. Hepatitis B, hepatitis C, syphilis, and HIV screening were negative. His renal function gradually declined over the following decade, resulting in ESRD. At 44 years of age, peritoneal dialysis was started for the treatment of ESRD, and an inguinal lymph node biopsy was performed to confirm the diagnosis, revealing the typical features of plasma cell-type MCD. Oral prednisolone (PSL) (10 mg/day) was initially administered; however, none of his clinical or laboratory parameters were fully resolved.

At 45 years of age, he was referred to our hospital for further evaluation and treatment including living-donor KTx. To histopathologically determine the primary cause of ESRD (i.e., IgA nephropathy, AA amyloidosis, or other causes) and to make a pathological diagnosis of his interstitial lung disease, renal and lung biopsies, respectively, were performed. Similar to the immunostaining findings of the inguinal lymph nodes, which showed significant deposits of IgA and IL-6 (Fig. 1a), lung biopsy specimens showed plasma cell proliferation with positive staining for IgA and IL-6 (Fig. 1b), and renal biopsy specimens showed predominant IgA deposition in the glomerular mesangium (Fig. 1c). These findings led us to the possible diagnosis of MCD with lung involvement complicated by ESRD associated with IgA nephropathy. Since treatment with oral PSL failed to suppress the disease activity of MCD, the therapeutic regimen was changed to intravenous TCZ ( $8 \mathrm{mg} / \mathrm{kg}$, every 2 weeks), which successfully controlled his MCD symptoms without the further exacerbation of his lung disease.

At 50 years of age, he had recurrent peritonitis and therefore decided to undergo KTx. Preoperatively, tacrolimus (TAC) and mycophenolate mofetil (MMF) were administered starting 5 days before transplantation. TAC was administered at $0.1 \mathrm{mg} / \mathrm{kg} /$ day, and the trough level was maintained at $8-12 \mathrm{ng} / \mathrm{mL}$ for the first few weeks after transplantation. MMF was administered at a dosage of $1000 \mathrm{mg}$ twice daily. In addition, induction with intravenous methylprednisolone (mPSL; 1 dose of $500 \mathrm{mg}$ ) and basiliximab (1 dose of $20 \mathrm{mg}$ ) therapy was administered on the day of transplantation. Basiliximab was also administered 4 days after transplantation. mPSL was gradually tapered to $40 \mathrm{mg}$ by the end of the first post-transplantation week and switched to an oral formulation. To prevent overimmunosuppression, TCZ was discontinued after transplantation. However, the extension of the interval between TCZ administrations was associated with a slight increase in the patient's CRP levels, but his constitutional symptoms did not reappear, and after the repeated administration of TCZ ( $8 \mathrm{mg} / \mathrm{kg}$, every 2 weeks), improvements in his CRP levels were immediately noted (Fig. 2).

The patient's IL-6 levels decreased dramatically after transplantation and are now maintained at 300-500 pg/ $\mathrm{ml}$ under immunosuppression with a triple-drug regimen (TAC, MMF and MPSL) with TCZ (Fig. 2). In addition, the patient experienced increases in albumin, haemoglobin and haematocrit (data not shown), along with normalized CRP and IgG (Fig. 2).

On post-operative days 20 and 238, a transplant renal biopsy was performed and showed no rejection or virus infection. To evaluate for the presence of anti-HLA antibodies, a single-antigen bead assay (LABScreen Single Antigen) was performed. DSA was negative both before transplantation and on post-operative day 239 .

At present, 17 months after $\mathrm{KTx}$, the patient remains asymptomatic, and the allograft function has been preserved without evidence of rejection. In addition, haematologic follow-up has not demonstrated any significant adverse effects of TCZ (such as dyslipidaemia or 


\section{A Lymph node biopsy}
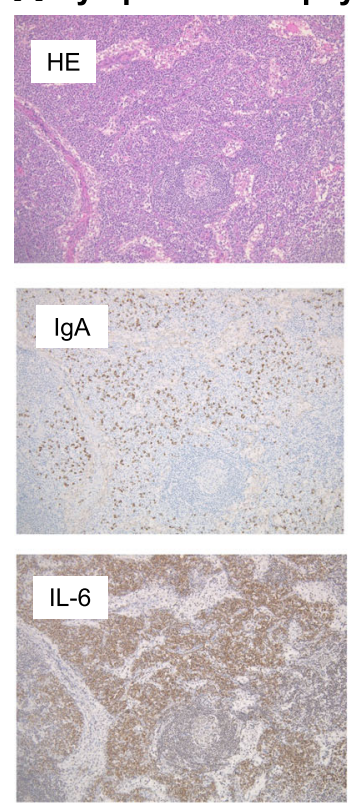

B Lung biopsy
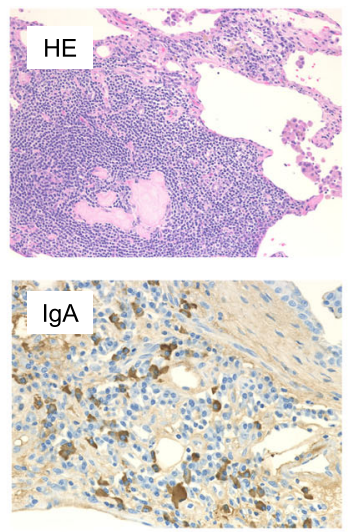

IL-6

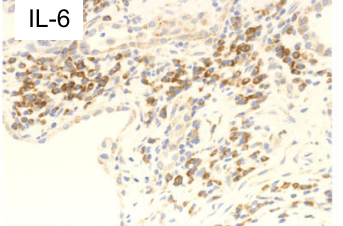

\section{Renal biopsy}
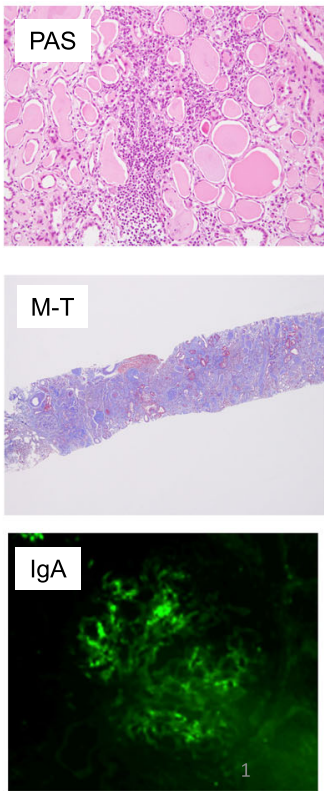

Fig. 1 Histology and immunostaining. Specimens obtained from the inguinal lymph node, lung and kidney. a A microscopic examination of the inguinal lymph node revealed interfollicular expansion with plasmacytosis, compatible with plasma cell-type MCD. Numerous IgA- and IL-6-positive cells were detected. $\mathbf{b}$ A lung biopsy shows prominent infiltration of plasma cells with IgA- and IL-6-positivity. c Periodic acid-Schiff staining and Masson trichrome staining show ESRD; the stained blue area is the fibrous tissue in the ESRD kidney. Immunofluorescence staining of a renal biopsy specimen shows granular staining for $\lg \mathrm{A}$ in the mesangium

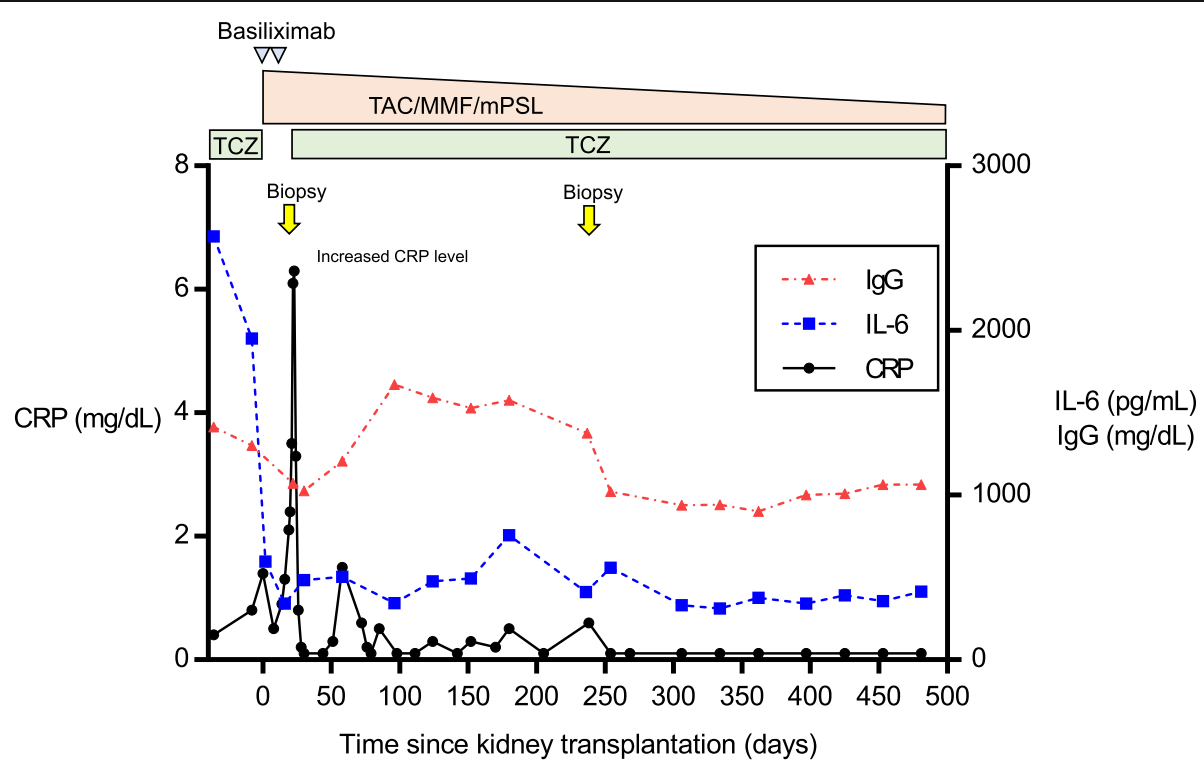

TAC: tacrolimus, MMF: mycophenolate mofetil, mPSL: methylprednisolone, TCZ: tocilizumab

Fig. 2 Clinical course after transplantation. Tapering of triple immunosuppression and sustained treatment with TCZ in an MCD transplant recipient. The IL-6 levels are currently maintained at 300-500 pg/ml, and the CRP and lgG levels are trending towards normal with no allograft rejection 
myelosuppression) or cytomegalovirus (CMV) infection events.

\section{Discussion and conclusions}

This case highlights two important clinical implications: MCD with ESRD can be successfully treated by KTx, and TCZ is useful for MCD and ensures a safe perioperative period during KTx.

In the past, the treatment of $\mathrm{CD}$ has included various therapeutic strategies, such as steroids, rituximab, and chemotherapy. However, since there is no standard therapy, treatment recommendations for $\mathrm{CD}$ can be difficult [3]. Recently, TCZ was shown to be effective for treatment and has been widely used in Japan since June 2005 [1, 2, 9, 10].

While renal involvement associated with MCD is rare, its manifestations can vary and include minimal disease changes, mesangial proliferative glomerulonephritis, AA amyloidosis, thrombotic microangiopathy (TMA) and IgA nephropathy $[4,5,11-14]$. In particular, IgA nephropathy associated with MCD is quite rare [11]. In our patient, in addition to the diagnosis of IgA nephropathy, numerous IgA-positive cells were detected in the lymph node and lung specimens. This result is consistent with those of a recent study, which found that the detection of increased immunohistochemical IgA expression can be useful for diagnosing CD [15]. Furthermore, ESRD as a complication of MCD has rarely been reported, and to our knowledge, only one report has described successful KTx for an MCD patient [6]. Murakami et al. reported an MCD patient with ESRD who underwent KTx. The patient developed acute rejection soon after KTx but was successfully treated with a steroid pulse, plasmapheresis, anti-CD3 and rituximab. The graft survival was 8 years, and the graft function remained excellent.

In patients with $\mathrm{CD}$, increased IL-6 and CRP levels have been noted $[1,2,12-14]$. CD exacerbation is correlated with the levels of both IL- 6 and CRP; IL-6 induces the synthesis of hepatic acute-phase reactants and increases CRP and IgG levels. In our patient, his IL-6 levels decreased dramatically after transplantation with induction therapy from $3480 \mathrm{pg} / \mathrm{ml}$ to $341 \mathrm{pg} / \mathrm{ml}$. We suspect that induction therapy might have dramatically decreased his IL-6 levels.

IL-6 is a major cytokine involved in the progression of B cells to IgG-secreting plasmablasts and finally to plasma cells. In addition, IL-6 stimulates Th17 cells, which mediate inflammation and allograft rejection. Recent studies have suggested that TCZ can inhibit antibody production and reduce inflammation by targeting the IL-6/IL-6R pathway [16-18]. Jordan et al. reported on the efficacy of TCZ in reducing anti-HLA antibodies and improving the graft survival in highly HLA-sensitized patients who were resistant to other desensitization strategies. Furthermore, they offered TCZ treatment to chronic antibody-mediated rejection patients in whom other treatment options had failed, and significant reductions in DSA and stabilization of renal function were seen at 2 years [7, 8].

Another study suggested that TCZ may be useful for preventing post-transplant graft-versus-host disease (GVHD) [19]. Kennedy et al. reported that the addition of TCZ to the standard GVHD prophylaxis for patients who had received allogenic stem-cell transplantation was safe and associated with a very low incidence of GVHD.

The long-term use of TCZ is optimal for the treatment of MCD; thus, treatment with TCZ may allow KTx patients to reduce or discontinue their conventional immunosuppression and mitigate related side effects, such as hypertension, dyslipidaemia, de novo diabetes, infection, and malignancies [20]. We therefore expect immunosuppression after KTx to also be effective for MCD.

In summary, we suggested a treatment protocol for renal replacement therapy using TCZ for MCD patients with ESRD. While the IL-6R monoclonal antibody TCZ was shown to be effective for treating MCD, it may also be used to reduce the risk of chronic antibody-mediated rejection and thereby possibly improve long-term graft survival $[7,8]$. Although the indications for elective KTx in MCD patients remain limited, continued success and reports of good outcomes may help establish KTx as an acceptable therapeutic option.

\section{Abbreviations}

CD: Castleman disease; CNI: Calcineurin inhibitor; DSA: Donor-specific antibody; ESRD: End-stage renal disease; GVHD: Graft-versus-host disease; IL-6: Interleukin 6; IL-6R: Interleukin-6 receptor; KTx: Kidney transplantation; MCD: Multicentric Castleman disease; MMF: Mycophenolate mofetil; MPSL: Methylprednisolone; PSL: Prednisolone; TAC: Tacrolimus; TCZ: Tocilizumab

\section{Acknowledgements}

The author would like to thank Dr. Hiroshi Harada and Dr. Hisako Saito for their careful patient management at Showa University Fujigaoka Hospital in Yokohama, Japan.

Availability of data and materials

All the data supporting our findings is contained within the manuscript.

\section{Authors' contributions}

MM designed and wrote the manuscript. KS, YO, MO, Kazuya Kinoshita, KT and $Y N$ treated the patient. Keiichi Kinowaki, KO, TF, TI and YS supplied the pathology reports. $Y U$ and $Y$ I supervised the transplant program and corrected the manuscript. All authors read and approved the final manuscript.

Ethics approval and consent to participate

Not applicable.

\section{Consent for publication}

Written informed consent was obtained from the patient to publish this case report and any accompanying images. A copy of the written consent is available for review by the editor of this journal.

Competing interests

The authors declare that they have no competing interests. 


\section{Publisher's Note}

Springer Nature remains neutral with regard to jurisdictional claims in published maps and institutional affiliations.

\section{Author details}

'Department of Surgery, Nephrology Center, Toranomon Hospital, 2-2-2 Toranomon, Minato-ku, Tokyo 105-8470, Japan. ${ }^{2}$ Nephrology Center, Toranomon Hospital, Tokyo, Japan. ${ }^{3}$ Department of Pathology, Toranomon Hospital, Tokyo, Japan. ${ }^{4}$ Okinaka Memorial Institute for Medical Research, Tokyo, Japan. ${ }^{5}$ Department of Pathology, Yokohama City University Graduate School of Medicine, Yokohama, Japan. 'Department of Pathology, Okayama University Graduate School of Medicine, Dentistry, and Pharmaceutical Sciences, Okayama, Japan.

Received: 11 June 2018 Accepted: 3 October 2018 Published online: 11 October 2018

\section{References}

1. Nishimoto N, Sasai M, Shima Y, Nakagawa M, Matsumoto T, Shirai T, Kishimoto T, Yoshizaki K. Improvement in Castleman's disease by humanized anti-interleukin-6 receptor antibody therapy. Blood. 2000;95(1): 56-61.

2. Nishimoto N, Terao K, Mima T, Nakahara H, Takagi N, Kakehi T. Mechanisms and pathologic significances in increase in serum interleukin-6 (IL-6) and soluble IL-6 receptor after administration of an anti-IL-6 receptor antibody, tocilizumab, in patients with rheumatoid arthritis and Castleman disease. Blood. 2008;112(10):3959-64.

3. Chan KL, Lade S, Prince HM, Harrison SJ. Update and new approaches in the treatment of Castleman disease. J Blood Med. 2016;7:145-58.

4. Xu D, Lv J, Dong Y, Wang S, Su T, Zhou F, Zou W, Zhao M, Zhang H. Renal involvement in a large cohort of Chinese patients with Castleman disease. Nephrol Dial Transplant. 2012;27(Suppl 3):iii119-25.

5. El Karoui K, Vuiblet V, Dion D, Izzedine H, Guitard J, Frimat L, Delahousse M, Remy P, Boffa JJ, Pillebout E, et al. Renal involvement in Castleman disease. Nephrol Dial Transplant. 2011;26(2):599-609.

6. Murakami K, Kobayashi T, Okubo K, Kamba T, Yoshimura K, Ogawa O. Successful renal transplantation for end-stage renal insufficiency developed in a patient with Castleman's disease. Transplant Int. 2013;26(7):e61-2.

7. Jordan SC, Choi J, Kim I, Wu G, Toyoda M, Shin B, Vo A. Interleukin-6, a cytokine critical to mediation of inflammation, autoimmunity and allograft rejection: therapeutic implications of IL-6 receptor blockade. Transplantation. 2017;101(1):32-44.

8. Choi J, Aubert O, Vo A, Loupy A, Haas M, Puliyanda D, Kim I, Louie S, Kang A, Peng A, et al. Assessment of tocilizumab (anti-Interleukin-6 receptor monoclonal) as a potential treatment for chronic antibody-mediated rejection and transplant Glomerulopathy in HLA-sensitized renal allograft recipients. Am J Transplant. 2017;17(9):2381-9.

9. Oshima $Y$, Hoshino J, Suwabe T, Hayami N, Yamanouchi M, Sekine A, Ueno T, Mizuno H, Yabuuchi J, Imafuku A, et al. Multicentric Castleman's disease associated with IgA vasculitis (Henoch-Schonlein purpura) responding well to tocilizumab: a case report. Clin Rheumatol. 2017;36(3):729-33.

10. Matsuyama M, Suzuki T, Tsuboi H, Ito S, Mamura M, Goto D, Matsumoto I, Tsutsumi A, Sumida T. Anti-interleukin-6 receptor antibody (tocilizumab) treatment of multicentric Castleman's disease. Intern Med (Tokyo, Japan) 2007:46(11):771-4

11. Komatsuda A, Wakui H, Togashi M, Sawada K. IgA nephropathy associated with Castleman disease with cutaneous involvement. Am J Med Sci. 2010; 339(5):486-90

12. Suneja S, Chidambaram M, Herzenberg AM, Bargman JM. Kidney involvement in multicentric castleman disease. Am J Kidney Dis. 2009;53(3): $550-4$

13. Ogita M, Hoshino J, Sogawa Y, Sawa N, Katori H, Takemoto F, Ubara Y, Hara S, Miyakoshi S, Takaichi K. Multicentric Castleman disease with secondary AA renal amyloidosis, nephrotic syndrome and chronic renal failure, remission after high-dose melphalan and autologous stem cell transplantation. Clin Nephrol. 2007;68(3):171-6.

14. Uthup S, Balachandran K, Ammal VA, Abdul Salam R, George J, Nair GM Leela M. Renal involvement in multicentric Castleman disease with glomeruloid hemangioma of skin and plasmacytoma. Am J Kidney Dis. 2006;48(2):e17-24
15. Manabe A, Igawa T, Takeuchi M, Gion Y, Yoshino T, Sato Y. Immunohistochemical analysis of IgA expression differentiates lgG4-related disease from plasma cell-type Castleman disease. Med Mol Morphol. 2017; 50(1):34-41.

16. Yeung MY, Gabardi S, Sayegh MH. Use of polyclonal/monoclonal antibody therapies in transplantation. Expert Opin Biol Ther. 2017;17(3):339-52.

17. Vo AA, Choi J, Kim I, Louie S, Cisneros K, Kahwaji J, Toyoda M, Ge S, Haas M, Puliyanda $D$, et al. A phase $1 / /$ trial of the Interleukin-6 receptor-specific humanized monoclonal (tocilizumab) + intravenous immunoglobulin in difficult to desensitize patients. Transplantation. 2015;99(11):2356-63.

18. Jordan SC, Choi J, Vo A. Kidney transplantation in highly sensitized patients. Br Med Bull. 2015;114(1):113-25.

19. Kennedy GA, Varelias A, Vuckovic S, Le Texier L, Gartlan KH, Zhang P, Thomas G, Anderson L, Boyle G, Cloonan N, et al. Addition of interleukin-6 inhibition with tocilizumab to standard graft-versus-host disease prophylaxis after allogeneic stem-cell transplantation: a phase 1/2 trial. Lancet Oncol. 2014;15(13):1451-9.

20. Madariaga ML, Spencer PJ, Shanmugarajah K, Crisalli KA, Chang DC, Markmann JF, Elias N, Cosimi AB, Sachs DH, Kawai T. Effect of tolerance versus chronic immunosuppression protocols on the quality of life of kidney transplant recipients. JCI Insight 2016;1(8).

Ready to submit your research? Choose BMC and benefit from:

- fast, convenient online submission

- thorough peer review by experienced researchers in your field

- rapid publication on acceptance

- support for research data, including large and complex data types

- gold Open Access which fosters wider collaboration and increased citations

- maximum visibility for your research: over $100 \mathrm{M}$ website views per year

At BMC, research is always in progress.

Learn more biomedcentral.com/submissions 